\title{
Will this trial change my practice? ABSORB II trial (a bioresorbable vascular scaffold versus drug-eluting stent in coronary disease)
}

\author{
Rodney De Palma1 ${ }^{1}, \mathrm{MB}, \mathrm{BS}$; Robert A. Byrne ${ }^{2}, \mathrm{MB}, \mathrm{BCh}, \mathrm{PhD}$; Robert-Jan van Geuns ${ }^{3}, \mathrm{MD}$;
} Thomas Pilgrim ${ }^{4}$ MD; Peter Jüni ${ }^{5}$, MD; Ibrahim Al-Rashdan ${ }^{6 *}$, MD

1.The Heart Hospital, University College London Hospitals UCLH, London, United Kingdom; 2. Deutsches Herzzentrum München, Technische Universität München and DZHK (German Centre for Cardiovascular Research), partner site Munich Heart Alliance, Munich, Germany; 3. Thoraxcenter, Erasmus Medical Center, Rotterdam, The Netherlands; 4. Department of Cardiology, Bern University Hospital, Bern, Switzerland; 5. Institute of Primary Health Care, University of Bern, Bern, Switzerland; 6. Department of Medicine, Faculty of Medicine, University of Kuwait and Chest Disease Hospital, University of Kuwait, Kuwait City, Kuwait

\section{The "Will this trial change my practice?"} sessions at PCR

The aim of the article is to capture the session at EuroPCR 2015, communicate the analysis of the trialists, and report the views expressed in the interactive discussion.

\section{Introduction to the session}

Bioresorbable stents (BRS) have been described as the fourth revolution in interventional cardiology. ABSORB II is the first randomised controlled clinical trial (RCT) comparing an everolimus-eluting BRS with a second-generation everolimus-eluting permanent metallic stent (DES) ${ }^{1}$. A dedicated interactive EuroPCR session subjected this study to focused scrutiny with the help of facilitators consisting of Ibrahim Al-Rashdan, Robert Byrne, Robert-Jan van Geuns, Thomas Pilgrim, and Peter Jüni. An initial poll of the audience indicated that the majority did not currently use BRS in their daily practice. An introduction and overview of the session was given by Robert Byrne who explained that the trial data would be evaluated and discussed together with a case study illustrative of some of the issues surrounding this novel platform which would be presented by Robert-Jan van Geuns: a patient with stable angina and ischaemia on non-invasive evaluation who had a BRS deployed in a severe stenosis within a calcified right coronary artery following rotational atherectomy. The patient was ineligible for ABSORB II based on the strict entry criteria but nevertheless was treated with BRS and entered into the ABSORB-EXPAND registry.

\section{Do we need a change in practice?}

Each iteration of permanent mechanical platform to treat ischaemic coronary stenosis has brought with it advantages as well as disadvantages including restenosis, neoatherosclerosis and stent thrombosis. The limitations of these platforms have driven the development of a drug-eluting resorbable platform that temporarily scaffolds a lesion during the phase of healing following implantation and then resorbs, leaving no residual material or nidus for stent thrombosis. Such a platform if efficacious and safe would be desirable.

*Corresponding author: Department of Medicine, Faculty of Medicine, University of Kuwait and Chest Disease Hospital, University of Kuwait, Kuwait City, Kuwait.E-mail: i.alrashdan@kuwaitheart.org 
Second-generation DES are currently the standard platform used for the majority of coronary lesions around the world. Their improved safety profile, ease of deliverability and data with millions of patient years of experience raises the bar for further device improvement to a high level. BRS is an innovative device that has the potential to change practice. Although currently use is limited, with approximately 100,000 patients having been treated to date according to the manufacturers, their use is increasing, and thus this first randomised trial comparing it to contemporary DES gives the interventional community an opportunity for reflection.

\section{Background}

Robert Byrne opened with an overview of the trial design, originally published in $2012^{2}$. The ABSORB II is an ongoing RCT of patients recruited from European and New Zealand centres with 2:1 enrolment (BRS:DES), allocation concealment, event adjudication blinding and intention-to-treat analysis. The primary endpoint, for which it is adequately powered, includes angiographically determined nitrate-inducible vasomotion of the target lesion segment at 36 months. Secondary endpoints include clinical (patient and device-orientated) events also at 36 months. In particular, patientrelated outcome measures in the form of angina evaluation with the validated Seattle Angina Questionnaire tool as well as noninvasive ETT evaluation were used. As the trial data were analysed at interim stages of six and 12 months, these recently published data along with the case illustration formed the basis of the ensuing discussion.

Robert-Jan van Geuns then continued with the case presentation of BRS. This was implanted into the distal RCA of a female patient with stable angina and ischaemia on non-invasive evaluation who was deemed not suitable for the ABSORB II RCT due to calcification and who was entered into a prospective registry. This case was illustrative of some of the issues surrounding the technology and inclusion of patients into the study and real-world use. For the ABSORB II RCT the entry criteria were restrictive and sought to include only simple coronary stenotic lesions: one or two de novo lesions in different vessels, no heavy calcification, $<48 \mathrm{~mm}$ lesion length, 2.25-3.8 $\mathrm{mm}$ diameter and successful predilatation (meaning a residual stenosis of $<40 \%$ ), and no use of adjunctive technology suggestive of a complex lesion such as rotational atherectomy and cutting balloon. Robert-Jan van Geuns went on to say that those not fulfilling the criteria in full but still considered eligible for a BRS are typically entered, and should be entered, into a prospective registry such as ABSORB- EXPAND or EXTEND. A further point was made that, if aggressive lesion preparation, as currently advocated by manufacturers, is adhered to, then even those in the registry have similar clinical outcomes to those of contemporary DES platforms.

Ibrahim Al-Rashdan, with reference to the case, pointed out an opposing view that the use of BRS whether in a trial or registry was inappropriate due to the heavy calcium burden and challenge of device trackability in such vessels.
Robert Byrne posed a practical question regarding whether, once a decision to deploy a BRS has been made, angiographic confirmation of scaffold expansion was sufficient or whether OCT was mandatory. The group confirmed that liberal use of OCT was best after deployment but that its use was not mandatory.

Thomas Pilgrim painted the background. There are currently sixteen products that have been developed, and these can be separated in broad terms into BRS made of a magnesium alloy and those made of PLLA. Several of these platforms elute a drug active against restenosis. To maintain comparable radial strength, their strut thickness is roughly double that of current metallic stents, making them bulky compared to DES. Their main appeal is their intrinsic resorbability over time. There are further potential physiological benefits that include improved vasomotion (both physiologically and pharmacologically induced), improved pulsatility (only demonstrated in porcine models so far), late lumen enlargement, and vulnerable plaque sealing (leading to the so-called "golden tube" on OCT). These encouraging parameters, however, need to translate into clinical benefit before being accepted as indicators of efficacy.

With this in mind, the latest registry data were summarised. ABSORB-EXTEND $(n=512)$ showed a respectable late lumen loss of $<0.5 \mathrm{~mm}$, mortality of $0.5 \%$ and infarction of $2.9 \%$, suggesting equivalent efficacy to contemporary DES ${ }^{3}$. GHOST-EU is the largest registry to date $(n=1,189)$ with $25 \%$ of lesions being bifurcations, reporting an overall $2.1 \%$ rate of definite or probable stent thrombosis ${ }^{4}$.

This background raises questions over the specific clinical settings best suited to BRS, whether strut thickness can be decreased further, and non-inferiority over current latest-generation drugeluting stents in terms of efficacy and, crucially, the safety of this platform. Large-scale randomised trials formed the consensus view of the group as to how best to resolve these questions, ABSORB II being the first of these and ABSORB III and IV due to follow on thereafter. ABSORB III is a larger RCT including 2,000 patients and more complex lesions (but still excluding calcified stenoses, as well as acute and chronic occlusions) with a similar surrogate non-clinical primary endpoint. ABSORB IV will include 3,000 patients and use angina and quality of life metrics as its primary endpoint as well as including cost-effectiveness analyses $^{5,6}$.

\section{Trial analysis}

Robert Byrne having given an overview, Peter Jüni provided an in-depth analysis of the study. As mentioned previously, the design of ABSORB II is that of a prospective randomised controlled clinical trial with 2:1 enrolment (BRS:DES), allocation concealment, patient blinding (although it was noted that 35 subjects in the BRS arm and five in the DES arm became unblinded), independent endpoint adjudication with blinding and an intention-to-treat analysis. The study was industry funded but with an independent academic steering group. The involvement of industry was raised as a concern as was the number of subjects 
unblinded. Although it is challenging to maintain a blinded study, it was posited that this should be the aim, particularly where endpoints can be affected by subjective interpretation. Nonetheless, the near complete follow-up of $>98 \%$ (with five subjects lost in the BRS arm and two in the DES arm) is a testament to the highquality conduct and rigour of the trialists and compares very well against RCTs in other disciplines where follow-up rates of circa $80 \%$ are commonplace.

The study is powered for superiority of BRS at three years (36 months) on the primary outcome of angiographically determined nitrate-induced vasomotion, and non-inferiority for in-stent late loss. All other endpoints are secondary and thus exploratory only. These endpoints include clinical (patient and device-orientated) events at 36 months and patient-related outcome measures (PROMS) in the form of angina evaluation with the Seattle Angina Questionnaire. Given the residual angina experienced by many following conventional metallic stent treatment, despite procedural success and no major adverse event, this is a potentially important endpoint.

The patient and lesion characteristics were described and demonstrated no significant differences between BRS and DES. The cohort is slightly younger than is typical of general interventional practice with a mean age of 61.5 (SD 10) and 60.9 (SD 10), respectively. The predominant presentation was stable angina but there were $20 \%$ with unstable angina, and it was posited that these should have been excluded. The lesions treated were predominantly intermediate (AHA class B1 and B2 lesions $>96 \%$ ) with almost no complex lesions (AHA class C).

Differences, however, were apparent with the procedural characteristics. The BRS group used lower mean inflation pressures compared to the DES group (14.23 vs. $15.03 \mathrm{~atm}$ ). Although the acute device and procedural success recorded did not differ, it was noted that the difference in inflation pressure was associated with a real difference in angiographic residual vessel diameter $2.64 \mathrm{~mm}$ (SD 0.4 ) vs. $2.8 \mathrm{~mm}$ (SD 0.3) and minimum luminal diameter $2.22 \mathrm{~mm}$ (SD 0.3) vs. $2.5 \mathrm{~mm}$ (SD 0.3).

The outcomes were subjected to scrutiny, in particular the use of PROMS. These have been used in other fields for many years and are now making their way into cardiovascular device trials. Self-reported angina was evaluated using the Seattle Angina Questionnaire (SAQ) tool. This consists of several domains - physical limitation, angina stability, medication use, treatment satisfaction, and disease perception. Although a difference was suggested by ETT data indicating ischaemic changes in favour of the BRS group ( $4.3 \%$ vs. $17.2 \%, \mathrm{p}=0.05$ ), there were no differences in SAQreported angina at 12 months. However, a post hoc exploratory analysis, excluding the first seven days following device deployment, of first occurrence and duration of recurrent or worsening angina was recorded. This analysis, in favour of BRS (16.4\% vs. $25.6 \%, p=0.015$ ), suggests that the cumulative rate of angina may be a better tool than examining angina at an isolated time point, and thus challenges the way we currently collect such data. This therefore warrants further investigation.
For harder clinical outcomes (composite of cardiac death, target vessel MI and clinically indicated target vessel revascularisation) (Figure 1), there was a trend towards more infarction in the BRS group ( $4.2 \%$ vs. $1.2 \%, \mathrm{p}=0.07)$ and minimally more stent thrombosis (three patients $[0.6 \%]$ vs. none, $p=1.0$ ). This tendency, reflected by greater troponin elevation in the BRS group, probably does not represent a real concern as examination of the device and procedure-related events recorded using contemporary definitions of periprocedural MI showed no difference.

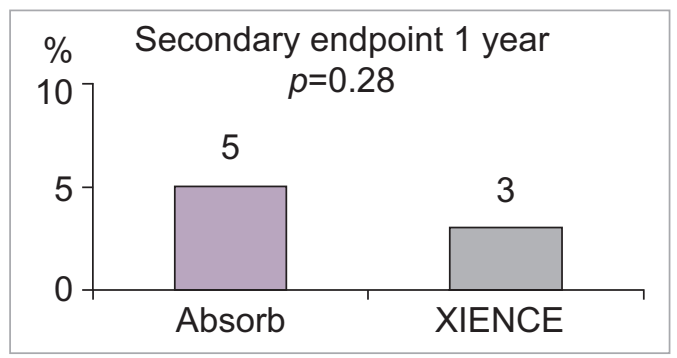

Figure 1. Cardiac death, all myocardial infarction, and clinically indicated target lesion revascularisation (major adverse cardiac events). Figure reproduced from the PCR Trials Book (C) 2015 Europa Digital \& Publishing).

Peter Jüni ended by reiterating the importance of blinded patients, especially with self-reported outcomes and excluding those who are unblinded. The question of the appropriateness of angiographic TLR as a performance measure for BRS was also raised on account of potential improvement in vasomotion properties.

\section{Session discussion (participants and facilitators)}

Ibrahim Al-Rashdan opened the discussion. It was felt that, despite ABSORB II, the signal of stent thrombosis in the largest contemporaneous registry had necessarily reduced use, and a 15-20\% market penetration ceiling for BRS was quoted. Importantly, the study interpretation was still supportive of BRS use within strict limits. Overall, the consensus was that a measure of benefit over a more appropriate length of time in a larger population representing daily practice was required.

One member of the audience agreed with the facilitators that BRS remains investigational. It was pointed out that operators needed to remember that BRS is more technically challenging to deploy than DES. This view was reinforced by Peter Jüni, with a reminder that the influence of the operator would be a major factor in the efficacy and safety of a device whenever a material was unforgiving as seems to be the case with BRS. A counter point made by RobertJan van Geuns was that, given the fact that we are still learning from our experience of the device, these concerns should not prevent continued use as part of registry or trial programmes. The use of interim analysis was questioned by Robert Byrne in terms of the validity of the final trial report at 36 months. Peter Jüni clarified 
that the data thus far were explorative and so would not endanger the final primary endpoint.

Ibrahim Al-Rashdan reminded the participants that there was a big difference between the real world and that of ABSORB II. Lesion preparation was key and, in particular, avoiding heavily calcified regions, keeping in mind that multiple projections were frequently necessary to determine this. Indeed Robert-Jan van Geuns had explained that the case study presented at the start was a complex lesion not eligible for ABSORB II and entered into a registry. Clinical and angiographic follow-up of the case revealed no adverse events at 12 months with further progression of more distal disease.

The challenges of device deployment are reflected in the continued evolution of the procedural implant recommendations, even following on from those in ABSORB II. More aggressive predilatation with a non-compliant balloon and 1:1 balloon:artery ratio is now advised. Cutting balloons and rotablation are now encouraged. A previously recommended overlap of 2-4 $\mathrm{mm}$ has now been superseded by a recommendation for no overlap. Post-dilatation is now highly recommended and mandatory if there is $>10 \%$ residual stenosis.

\section{Major views expressed regarding BRS use}

- Lesion selection and preparation using the latest recommendations is paramount.

- ABSORB II interim data are exploratory only; longer-term follow-up and larger population experience are required.

- Continued use is justified only as part of a trial or established registry programme.

- Stent thrombosis, once the data on MI were analysed, did not indicate a concern.

- The evaluation of angina as an efficacy endpoint for these coronary devices is still evolving, and the relevance of new measures is not entirely clear.

\section{Final audience poll}

For the minority who use BRS, the trial experience thus far would influence them to adhere to the latest technical recommendations. However, a poll of the majority not implanting revealed a consensus that the data were not sufficient to alter their practice. In the light of this, the final results of ABSORB II as well as the larger ABSORB III and IV are eagerly awaited.

\section{Conclusion}

Ibrahim Al-Rashdan concluded the session by reiterating the importance of sticking to rigorous selection criteria and the latest recommended implantation technique for BRS at all times. A signal of stent thrombosis, while not substantiated in this study thus far, may have occurred in registry data due to suboptimal implantation techniques, particularly in complex lesion subsets. The results of PROMS as well as post hoc angina burden analysis and what they mean should be interpreted with caution as far as BRS are concerned.

Nevertheless, the BRS device and concept remain exciting and, although currently there are no overwhelming data to support real-world practice change, "we must not throw the baby out with the bathwater".

\section{Summary box}

\section{Major arguments for a change in practice}

- Residual angina can affect a significant proportion of patients treated with conventional stent technology despite good procedural and major clinical outcome. Vascular scaffolds may lead to reduced angina burden.

- Equivalent MACE between DES and BRS.

- No signal of stent thrombosis if selection criteria and recommended implantation technique used.

\section{Major arguments against change}

- ABSORB II has not reported final results and so the currently released interim data on secondary endpoints at six and 12 months are exploratory only. Ultimately, the primary endpoint will be a non-clinical surrogate of angiographically determined nitrate-induced vasodilatation at 36 months.

- Real-world adherence to a more rigorous selection and implantation for current BRS may be challenging. Stent thrombosis in observational series may relate more to the implantation technique than to the scaffold. Nevertheless, much greater observational data are needed to prove safety.

- Interpretation of angina evaluation as an efficacy outcome in coronary device trials is evolving. Angina evaluation so far suggests no benefit, and exploratory analysis of total angina burden is post hoc.

\section{Conflict of interest statement}

The authors have no conflicts of interest to declare.

\section{Session format}

Chairperson: I. Al-Rashdan

Co-chairperson: R. Byrne

Participant: R. De Palma

Introduction: the trial headlines - R. Byrne

A case: how should I treat? - R-J. van Geuns

Discussion: what is common practice?

What was known before ABSORB II - T. Pilgrim

Trialist's review: methods, results, conclusion - P. Jüni

Discussion and audience interaction

Case conclusion: how does this apply to my practice?

R-J. van Geuns

Discussion and audience interaction

Session evaluation and key lessons - I. Al-Rashdan

\section{References}

1. Serruys PW, Chevalier B, Dudek D, Cequier A, Carrié D, Iniguez A, Dominici M, van der Schaaf RJ, Haude M, Wasungu L, Veldhof S, Peng L, Staehr P, Grundeken MJ, Ishibashi Y, GarciaGarcia HM, Onuma Y. A bioresorbable everolimus-eluting scaffold versus a metallic everolimus-eluting stent for ischaemic heart disease 
caused by de-novo native coronary artery lesions (ABSORB II): an interim 1-year analysis of clinical and procedural secondary outcomes from a randomised controlled trial. Lancet. 2015;385:43-54.

2. Diletti R, Serruys PW, Farooq V, Sudhir K, Dorange C, Miquel-Hebert K, Veldhof S, Rapoza R, Onuma Y, GarciaGarcia HM, Chevalier B. ABSORB II randomized controlled trial: a clinical evaluation to compare the safety, efficacy, and performance of the Absorb everolimus-eluting bioresorbable vascular scaffold system against the XIENCE everolimus-eluting coronary stent system in the treatment of subjects with ischemic heart disease caused by de novo native coronary artery lesions: rationale and study design. Am Heart J. 2012;164:654-63.

3. Abizaid A, Ribamar Costa J Jr, Bartorelli AL, Whitbourn R, van Geuns RJ, Chevalier B, Patel T, Seth A, Stuteville M, Dorange C, Cheong WF, Sudhir K, Serruys PW. The ABSORB
EXTEND study: preliminary report of the twelve-month clinical outcomes in the first 512 patients enrolled. EuroIntervention. 2015;10:1396-401.

4. Capodanno D, Gori T, Nef H, Latib A, Mehilli J, Lesiak M, Caramanno G, Naber C, Di Mario C, Colombo A, Capranzano P, Wiebe J, Araszkiewicz A, Geraci S, Pyxaras S, Mattesini A, Naganuma T, Münzel T, Tamburino C. Percutaneous coronary intervention with everolimus-eluting bioresorbable vascular scaffolds in routine clinical practice: early and midterm outcomes from the European multicentre GHOST-EU registry. EuroIntervention. 2015;10:1144-53.

5. ABSORB III Randomized Controlled Trial (RCT) (ABSORBIII). https:/clinicaltrials.gov/ct2/show/NCT01751906

6. Absorb IV Randomized Controlled Trial. https://clinicaltrials.gov/ct2/show/NCT02173379 